\title{
Beyond the learning curve: a review of complex cases in robotic thoracic surgery
}

\author{
Travis C. Geraci, Joshua Scheinerman, David Chen, Amie Kent, Costas Bizekis, Robert J. Cerfolio, \\ Michael D. Zervos
}

Department of Cardiothoracic Surgery, New York University Langone Health, New York, NY, USA

Contributions: (I) Conception and design: MD Zervos; (II) Administrative support: MD Zervos; (III) Provision of study materials or patients: TC Geraci, J Scheinerman, D Chen; (IV) Collection and assembly of data: TC Geraci, J Scheinerman, D Chen; (V) Data analysis and interpretation: TC Geraci, J Scheinerman, D Chen; (VI) Manuscript writing: All authors; (VII) Final approval of manuscript: All authors.

Correspondence to: Travis C. Geraci, MD. Department of Cardiothoracic Surgery, New York University Langone Health, $5301^{\text {st }}$ Avenue, Suite $9 \mathrm{~V}$, New York, NY 10016, USA. Email: travis.geraci@nyulangone.org.

\begin{abstract}
The number of thoracic surgery cases performed on the robotic platform has increased steadily over the last two decades. An increasing number of surgeons are training on the robotic system, which like any new technique or technology, has a progressive learning curve. Central to establishing a successful robotic program is the development of a dedicated thoracic robotic team that involves anesthesiologists, nurses, and bed-side assistants. With an additional surgeon console, the robot is an excellent platform for teaching. Compared to current methods of video-assisted thoracoscopic surgery (VATS), the robot offers improved wristed motion, a magnified, high definition three-dimensional vision, and greater surgeon control of the operation. These advantages are paired with integrated adjunctive technology such as infrared imaging. For pulmonary resection, these advantages of the robotic platform have translated into several clinical benefits, such as fewer overall complications, reduced pain, shorter length of stay, better postoperative pulmonary function, lower operative blood loss, and a lower 30-day mortality rate compared to open thoracotomy. With increased experience, cases of greater complexity are being performed. This review article details the process of becoming an experienced robotic thoracic surgeon and discusses a series of challenging cases in robotic thoracic surgery that a surgeon may encounter "beyond the learning curve". Nearly all thoracic surgery can now be approached robotically, including sleeve lobectomy, pneumonectomy, resection of large pulmonary and mediastinal masses, decortication, thoracic duct ligation, rib resection, and pulmonary resection after prior chest surgery and/or chemoradiation.
\end{abstract}

Keywords: Robotic surgery; thoracic; outcomes; learning-curve; technique

Submitted Feb 20, 2020. Accepted for publication Aug 05, 2020.

doi: $10.21037 /$ jtd-2019-rts-05

View this article at: http://dx.doi.org/10.21037/jtd-2019-rts-05

\section{Introduction}

Over the last two decades, the use of robotic technology has accelerated in thoracic surgery. The continued growth of robotics has evolved with the improvement of machinery combined with an educated patient population seeking innovative care. Utilizing minimally invasive techniques, a robotic approach provides the surgeon with greater overall control of the operation via precise, wristed manipulation of multiple instruments, a magnified high-definition camera, seated ergonomics, and the option of a tandem teaching console. A robotic approach has been applied to nearly all procedures in the chest, including surgery of the lung, esophagus, and mediastinum with outstanding short-term results published by high-volume robotic surgeons (1).

Although it is established that robot surgery translates to excellent outcomes for experienced surgeons, the process of transitioning from open surgery or video-assisted thoracoscopic surgery (VATS) to a robotic approach 
requires energy, time, and humility. The learning curve in robotic thoracic surgery has been studied and requires stepwise experience with cases of gradual complexity. Overall, operative and postoperative outcomes after robotic thoracic surgery have been shown to improve in consecutive cohorts of patients.

In this paper, our intention is to look beyond the learning curve and present an array of cases that a robotic surgeon may encounter after mastery of standard procedures (such as pulmonary lobectomy). Compared to VATS, and in many cases even open surgery, the robotic system provides superior optics and maneuverability in the confined anatomy of the chest, permitting precision surgery particularly adept for engaging challenging pathologies. Our aims are twofold: to illustrate the diversity and complexity of thoracic surgery that can be performed on a robotic platform and secondly, to offer procedural guidance in these cases, learned from our experience in robotic surgery.

\section{The robotic learning curve}

The development of a thoracic robotic program is dependent on the entire surgical team, institutional culture, and a cohort of dedicated surgeons. The process of transitioning to robotics has been shown to be safe and feasible, and can be achieved in a relatively short period of time. As detailed by Cerfolio and colleagues, the optimal transition process begins with on-line and on-site training, followed by attendance at training courses and observational visits to the operating rooms of experience robotic surgeons (2). Once performing surgery, surgeons must assure dedicated operating room time on the robot to build consistency and efficiency in the robotic team and accumulate surgeon experience.

As with any new procedure or technology, there is a learning curve associated with robotics. While there is no definitive inflection point, perioperative outcomes in robotic thoracic surgery have been shown to improve with increasing surgeon and institutional experience. Estimates of a numeric learning curve vary widely (reports diverge from 15 to 200 cases for robotic lobectomy) and is dependent on case complexity and the prior experience of the surgeon. Reviewing robotic lobectomy in a single-surgeon series, for example, Baldonado and colleagues reported steady reductions in operative time, blood loss, chest tube duration, overall hospital stay and decreased rate of complications with increasing cumulative operative experience (3). There were a total of 6 vascular injuries, all of which occurred in an early cohort of cases. In a similar study reviewing the learning curve for robotic segmentectomy, Zhang and colleagues, assessed a single surgeon experience over 2 years, divided into three phases: the first 21 cases, cases 22-46, followed by cases 47-104 (4). The authors performed a perioperative and "clinicopathologic" outcome assessment to measure successful performance of segmentectomy over time. Operative time and estimated blood loss (EBL) decreased after the initial phase, however, other perioperative outcomes were similar across the three cohorts. A risk-adjusted analysis was performed to account for a number of clinical variables such as age, tumor size, and surgical difficulty, revealing that surgical competence was achieved in the second cohort, after approximately 40 cases.

It can be useful to classify operation types by level of difficulty, and first perform "beginner" level operations with the robotic approach. "Beginner" or "Level I" operations include pleural biopsy, wedge resection, resection of a pulmonary nodule $<3 \mathrm{~cm}$, lymph node removal, esophageal or posterior mediastinal cyst resection, and anterior mediastinal cyst/mass resection $<3 \mathrm{~cm}$. After mastery of these cases, Level II cases include thymectomy, resection of posterior mediastinal tumors, diaphragm plication, leiomyoma of mid-esophagus and chest wall resection. Level III cases include segmentectomy, lobectomy, pulmonary sleeve resection, and esophagectomy. In our experience with lobectomy and segmentectomy, we find that starting with lower lung resections is the safest strategy, as they tend to be most forgiving in regard to difficulty of resection.

Uniquely, many surgeons transition to robotic surgery after proficiency in VATS or open thoracic techniques, thereby potentially accelerating the learning curve of robotics with prerequisite knowledge of the anatomy and general conduct of the operation. New knowledge and skills are required for surgeons transitioning to robotics, such as instrument exchange and control, comfort with distance from the patient bedside, and maturation of visual cues to guide tissue handling. In review of the Society for Thoracic Surgery (STS) General Thoracic Database, Feczko and colleagues compared the transitioning process to robotic lobectomy among "de novo", open-experienced, and VATS-experienced surgeons (5). Initial and sustained proficiency related to major morbidity was lower in the de novo group, but similar among open and VATS surgeons. After approximately 20 cases, the majority of all surgeons achieved proficiency. Operative efficiency was difficult for all surgeons, with the majority of surgeon's requiring greater 
than 250 minutes of operative time to complete a robotic lobectomy. Interestingly, de novo surgeons performed faster on the robot than their open or VATS counterparts. In conclusion, the authors posited that for robotic lobectomy, outcomes improve significantly after 20 cases, but that the learning curve extends to 50 cases when accounting for operative time. The authors report a non-uniform effect of robotic proficiency with prior thoracic experience.

Simulation modules, both video-based and on a training console, can help accelerate the learning process by optimizing control and understanding of the robotic system prior to performing surgery. Basic training courses and proctored cases can further help maximize proficiency and efficiency. Optimization of technique and detailed conduct of the operation for more advanced cases can be improved with surgeon-proctored cases either in person, or via video conferencing technology. There is no official evidence-based credentialing process for robotic thoracic surgery. Proficiency parameters are typically dependent on individual institutions and/or departments and are therefore, highly variable.

In our experience, there is no true robotic learning curve. We find that operative and perioperative outcomes continue to improve, even after years of experience. More important, is the willingness of the robotic team and surgeon to identify areas of inefficacy and error and to promote continual change and improvements to technique and operative conduct. Equally, there is no level of mastery that cannot be advanced by either preoperative or intraoperative consultation from a partner surgeon. We encourage the use of colleagues to discuss approaches to challenging cases and promote collaborative efforts among surgeons in our operating rooms.

\section{Managing complications-conversion to open}

It is critical to understand how to manage complications during robotic thoracic surgery. Equally, surgeons must know when to abandon the robotic approach and covert to an open thoracotomy. Given the surgeon's physical distance from the patient's bedside and the unique risks for iatrogenic trauma due to lack of haptic feedback from the robotic instruments, injuries to the airways and vasculature may occur. In 2019, Cao and colleagues published a retrospective multi-institutional study reviewing the incidence and management of intraoperative catastrophes in robotic pulmonary resection (6). In the review, of which $85 \%$ of enrolled patients underwent robotic lobectomy, 35 patients $(1.9 \%)$ experienced an intraoperative catastrophe, defined as events necessitating emergency thoracotomy or a second unplanned major surgical procedure. The three operations most commonly resulting in a catastrophic event were left-upper lobectomy, bilobectomy, and pneumonectomy. The most common catastrophic events were injury to the pulmonary artery $(80 \%)$ or pulmonary vein $(6 \%)$. In the review, the most common contributing factor noted by the surgeon in the event of an injury to the pulmonary artery was adherent, calcified, or granulomatous hilar lymphadenopathy, particularly severe in the setting of neoadjuvant therapy.

There is a step-wise approach to dealing with pulmonary arterial or venous injuries during robotic surgery, known colloquially as "the 7 P's:" preparation, pressure, patience, poise, products, partner, and prolene. First, the surgeon must be ready for the possibility of an iatrogenic injury before starting the case. A thoracotomy tray and blood products should be available. When the injury occurs, the initial move is to apply pressure. An attempt can be made (in experienced hands) at robotic suture repair once proximal control is obtained. However, in most cases, we recommend early conversion to open thoracotomy for pulmonary artery injuries with direct repair of the injury with maximal exposure. The assistant should maintain pressure with a handheld sponge on an instrument while the surgeon makes the thoracotomy.

\section{Establishing an advanced robotic program}

Robotic thoracic surgery requires coordination from a number of team members in the operating room: a dedicated bedside assistant, an experienced anesthesiologist, and knowledgeable surgical technologists and nurse circulators. From the outset, the success of the robotic program, from the granular case-by-case level to the maturation of the program, is dependent on a functional team. It is important to observe in each case what is working and what isn't with respect to each team member's responsibilities and go over the successes and failures of each case with each team member on a daily basis-only then can the entire team improve, leading to optimal patient outcomes.

\section{Optimizing bedside assistance}

A skilled bedside assistant is critical for fluid instrument exchange and maneuvers at the robot-patient interface. 


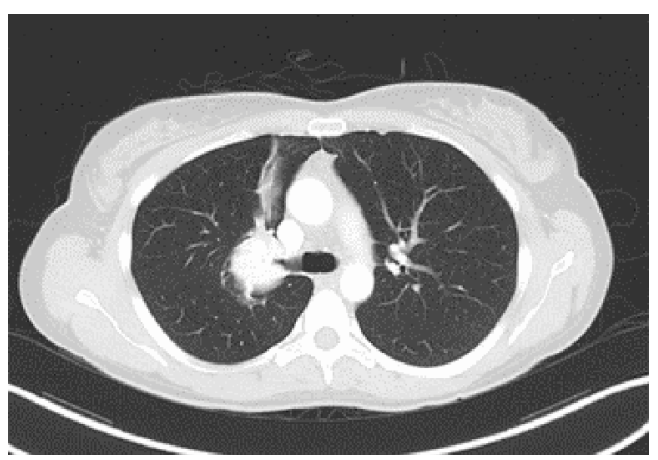

Figure $1 \mathrm{CT}$ chest, tumor obstructing the orifice of the right upper lobe bronchus. CT, computed tomography.

Central to success of bedside assistance is having a dedicated assistant that understands the nuances of the surgeon and has shared familiarity of the conduct of the operation. Equally, clear communication between the console and bedside assistant is crucial to safe and efficient surgery. We utilize a read-back system where directions and exchanges are repeated to assure clarity of instruction, particularly equipment motion external to the robotic camera, such as adjusting the robotic arms or loading instruments.

Certain bedside techniques help maximize efficiency during robotic thoracic surgery. For example, it is helpful to position the robot perpendicular to the patient and drive in directly, avoiding time-consuming maneuvering of the robotic cart. After initial port placement, the instrument arms should be reduced from the chest to the most distal marker (thin black line) and the camera should be reduced to just below the fascial level. These maneuvers allow the greatest instrument range of motion and generate the widest field of view. We utilize an AirSeal system (ConMed, Utica, NY, USA) to insufflate the chest with carbon dioxide, thereby depressing the lung tissue and diaphragm to generate space. The AirSeal system also evacuates smoke generated by tissue cautery, which maintains a clear field of view, thereby limiting camera exchanges.

\section{Teaching on the robot}

The robotic system includes an optional second console, which is a particularly effective training tool. The teaching console shares the exact field of view with the trainee as the operating surgeon. Equally, the console equipment is the same and control of the instruments can be exchanged fluidly. At our institution, residents and fellows are required to complete a robotic curriculum prior to working on the robotic console. The training includes a series of video presentations, simulation modules, and experience in bedside assisting.

\section{Challenging cases in robotic thoracic surgery}

\section{Sleeve lobectomy}

The use of the robotic system to perform bronchial sleeve resection and reconstruction for patients with centrally located pathology has been reported in a small number of single-surgeon case series (Figure 1). These reports consistently conclude that the procedure as both feasible and safe in select patients. The largest single-institutional series of 67 patients undergoing robotic bronchial sleeve lobectomy reported a $21 \%$ morbidity rate, no conversions to open, and no mortalities within 90 days (7). This series reported a mean duration of surgery of 167 minutes with a bronchial anastomosis time of 21 minutes. Sleeve lobectomy has also been shown to be oncologically comparable to pneumonectomy, with reduced postoperative morbidity, preservation of pulmonary function, and superior quality of life (8). Morbidity has been shown to be dependent on preoperative comorbidities, older age, and surgeon experience. Local recurrence rates are reported between $2-10 \%$.

In our experience, a 4-arm totally endoscopic approach is utilized with an assist port and bedside assistant (9). The conduction of the operation begins with a complete lymph node dissection. The target bronchus is mobilized with attention to avoiding unnecessary peribronchial dissection thereby preventing devascularization of the bronchus. To avoid stricture of the bronchial anastomosis, it is equally important to avoid undue tension. Bronchoscopy is performed to identify the margins of resection, which can be observed simultaneously on the robotic console. Before a bronchotomy is performed, it is important to reduce the inspired oxygen to $20 \%$ or less to reduce the risk of airway fire. The bronchus is entered sharply, starting most commonly at the distal margin, which allows the pathologic lobe to fall away from the surgical field. Curved robotic scissors or Potts scissors can be used. A proximal bronchotomy is then performed. A minimum of $1 \mathrm{~cm}$ of macroscopically healthy bronchus is optimal. A bronchial margin is excised and sent to pathology as a frozen section to assure negative microscopic margins.

The bronchial anastomosis can be completed with either a 


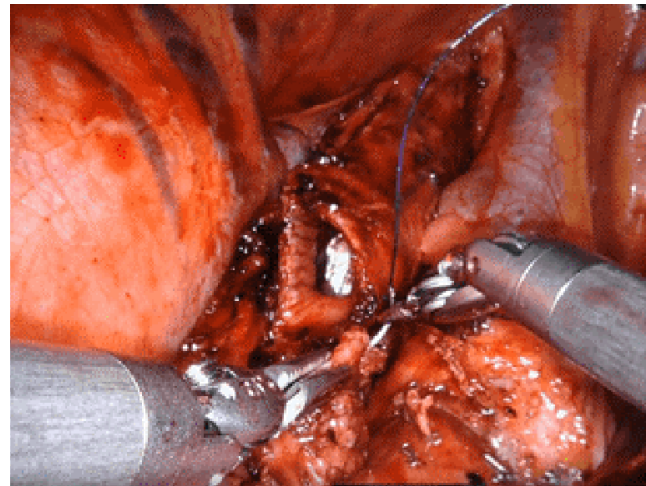

Figure 2 Bronchial anastomosis, right upper lobe sleeve lobectomy. Case: a 42-year-old woman was found to have an endobronchial carcinoid of the right upper lobe, obstructing the entire bronchus, with associated parenchymal volume loss and atelectasis. A robotic right upper lobe sleeve lobectomy with mediastinal lymphadenectomy and pleural wrap was performed.

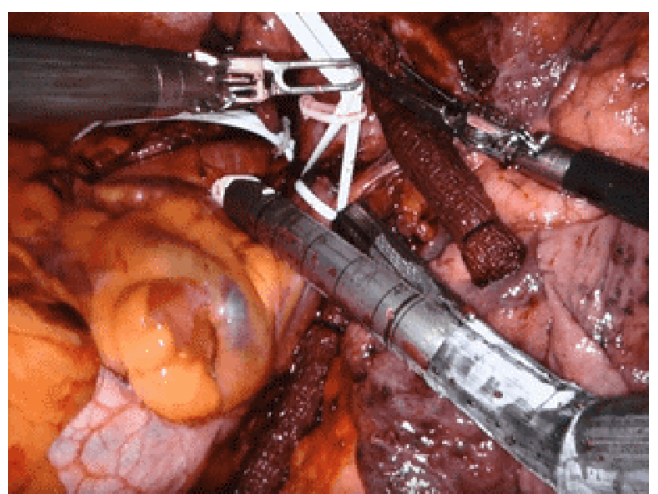

Figure 3 Left intrapericardial pneumonectomy, stapling of the common pulmonary vein (background: vessel loop control of the pulmonary artery). Case: a 79-year-old man was found to have a large left hilar lung mass with compression of the left atrium and pulmonary artery. Biopsy revealed leiomyosarcoma, which was removed by left robotic pneumonectomy with intrapericardial control of the hilar vessels.

completely robotic approach (our preference) or via a hybrid assist incision. A completely running technique or a combination of running and interrupted sutures have both been reported. Vicryl, PDS, Prolene and Stratafix sutures have all been used successfully. Our bronchial anastomosis is performed completely robotically with two running 3-0 Stratafix sutures (Figure 2). Usually one suture is not long enough to run the entire anastomosis. The anastomosis starts out-to-in on the distal bronchus connecting the inferior most aspects of the cartilaginous airways. The suture is run anteriorly along the cartilaginous portion of the airway. When complete, the second suture is run posteriorly down the membranous portion of the airway. The membranous portion is completed last to avoid tearing. A leak test is performed to assure technical closure of the anastomosis. A pleural wrap is not obligatory, but can be used to cover the bronchial anastomosis to promote healing.

\section{Pneumonectomy}

Minimally invasive pneumonectomy for lung cancer has been shown to be feasible and safe, supported in the literature by a number of small institutional series (10). Limited data are available regarding a robotic approach to pneumonectomy. Pneumonectomy often follows induction therapy and resection can be difficult due to adhesions and fibrosis of hilar lymph nodes. Careful selection of patients for a minimally invasive approach is critical to achieve excellent postoperative outcomes, particularly assuring the patient has adequate pulmonary function.

From a technical perspective, port placement for robotic pneumonectomy is the same as lobectomy, typically at the $8^{\text {th }}$ intercostal interspace. Preoperative decision-making is required to determine the operative plan, particularly regarding whether an intrapericardial approach is required. An intrapericardial approach allows surgery through clear tissue planes and is useful when the hilum is hostile of when the tumor location is central, which foreshortens the pulmonary vessels, making dissection difficult. Approaching the right or left lung, dissection begins at the anterior mediastinal pleura to expose the pulmonary hilum. The pericardium is typically opened below the phrenic nerve and dissection proceeds with sequential ligation and transection of the inferior pulmonary vein, superior pulmonary vein, pulmonary artery and lastly, the bronchus (Figure 3).

Leaving a long bronchial stump increases the risk of dehiscence leading to bronchopleural fistula. Attention, therefore, must be given to assuring a short proximal bronchial stump. In the right chest, this is performed more easily than in the left chest, due to obstruction of the aortic arch. Before stapling the bronchus, the planned staple line can be clamped and intraoperative bronchoscopy performed to assess the bronchial length. Additionally, the stapling angle of the bronchus should be perpendicular, thereby opposing the anterior and posterior bronchial walls. 


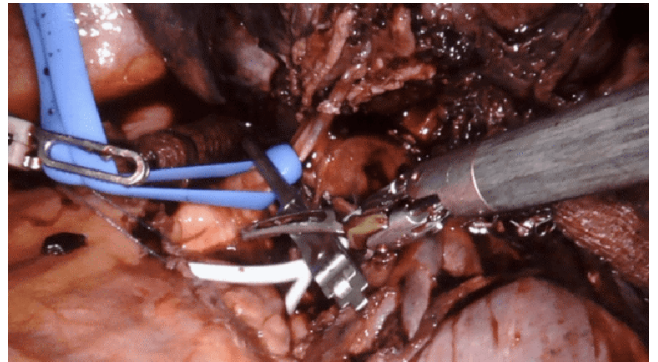

Figure 4 Intrapericardial control of the pulmonary artery; proximal via a bulldog clamp and vessel-loop (blue); distal control with a vessel loop (white). Case: a 54-year-old man was found to have a squamous cell lung cancer of the left upper lobe that invaded the posterior mediastinum, compressing the pulmonary artery to the left upper lobe. The patient underwent induction chemotherapy followed by robotic left upper lobectomy. Due to the tumor location compressing the pulmonary artery and extension into the mediastinum, proximal control of the pulmonary artery was obtained in the pericardium to reduce the risk of bleeding and to assure an $\mathrm{R} 0$ resection.

This technique promotes healing and decreases the risk of developing a bronchopleural fistula. In the left chest, we use the assist port to introduce the stapler, encircle the bronchus, and direct the tip of the stapler above the arch of the aorta. This ensures the proper stapling angle, parallel to the descending aorta, and seats the stapler low on the bronchus. A muscle-flap can be used to cover the bronchial stump, but is rarely necessary, particularly in the left chest as the bronchus retracts under the aortic arch.

Usually, closing the pericardium is not necessary. If a large section of pericardium is excised, however, a pericardial patch can be placed to prevent cardiac herniation. The pneumonectomy specimen is removed via an EndoCatch bag from the anterior port. An incision connecting adjacent ports can be made to accommodate larger lungs, but a decompressed lung rarely requires an incision greater than $5 \mathrm{~cm}$. If the specimen does not extract easily, the lung can be divided intracorporeally as permitted without disrupting the tumor. Rib-spreading is not required for extraction.

\section{Intrapericardial control of the pulmonary artery}

Intrapericardial control of the pulmonary vessels, particularly the pulmonary artery, is indicated in scenarios in which the vasculature is difficult to dissect, increasing the risk of injury and subsequent bleeding. Cases with large tumors that foreshorten the pulmonary artery, or where the hilar tissue planes are obliterated (such as after induction therapy for cancer, or during inflammatory cases where lymph nodes are adherent to pulmonary artery branches), it is useful to obtain proximal control of the hilum in the pericardium.

Intrapericardial control of the pulmonary artery can be achieved both proximally and distally, either with a vessel loop and/or bulldog clamp (Figure 4). Alternatively, for distal control, the inferior pulmonary vein can be clamped. A flex aortic cross-clamp can also be introduced externally to clamp the vessels. After proximal and distal control are obtained, dissection of the hilum can proceed with the knowledge and security that potential bleeding can be managed.

\section{Large pulmonary mass ( $>5 \mathrm{~cm}$ )}

Limited data has been published regarding the feasibility of robotic pulmonary resection for large lung lesions, typically defined as $5 \mathrm{~cm}$ or greater (Figure 5). Early studies in robotic thoracic surgery excluded large lesions, reserving these masses for resection via thoracotomy. With greater surgical experience and evolution of robotic technology, however, a greater number of large pulmonary lesions are being resected. The robotic system is adept at resection of large lesions, with the ability to efficiently retract tissue with a stable robotic arm. Admittedly, after a minimally invasive approach, extension of a port site is required at the end of the case for specimen removal.

In our experience, when compared to pulmonary resection for lesions less than $5 \mathrm{~cm}$, resection of large masses requires increased operative time, results in greater EBL, and has a higher rate of conversion to open. Equally, manipulating the lung and creating the appropriate exposures are more difficult due to limited space in the chest. Dividing pulmonary vascular branches should be done with care given the likelihood of foreshortened branches and/or greater tension due to displacement by the large mass.

\section{Large anterior mediastinal mass (>4 cm)}

The safety and feasibility of robotic thymectomy for large thymomas has been established in the literature. In a comparison study, robotic and transsternal thymectomy were evaluated from a single-institution database review (11). 

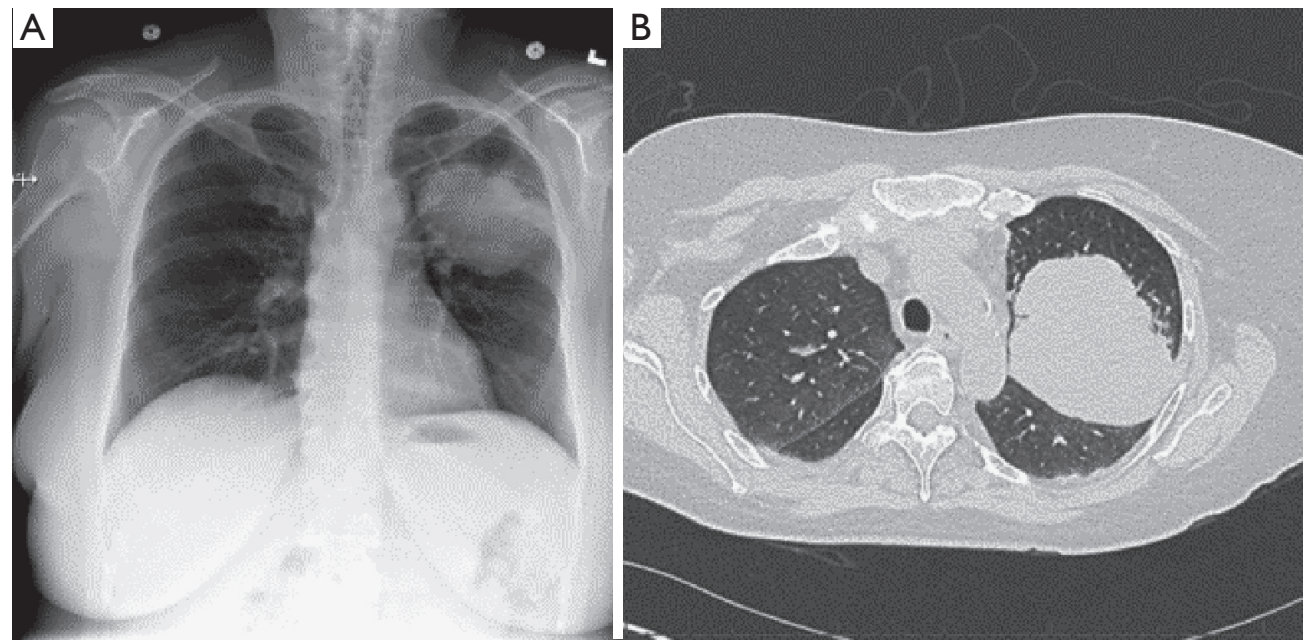

Figure 5 Large left upper lobe mass. (A) CXR; (B) CT scan of the chest. Case: a 53 -year-old female with an 8.6-cm mass in the left upper lobe. Transthoracic biopsy revealed adenocarcinoma and an endobronchial ultrasound (EBUS) did not demonstrate cancer in the mediastinal lymph node stations 2, 4, and 7 (T4N0M0). Post neoadjuvant chemotherapy, the patient underwent robotic left upper lobectomy and superior segmentectomy of the left lower lobe (superior segment was found to be involved with tumor). CXR, chest X-ray; CT, computed tomography.

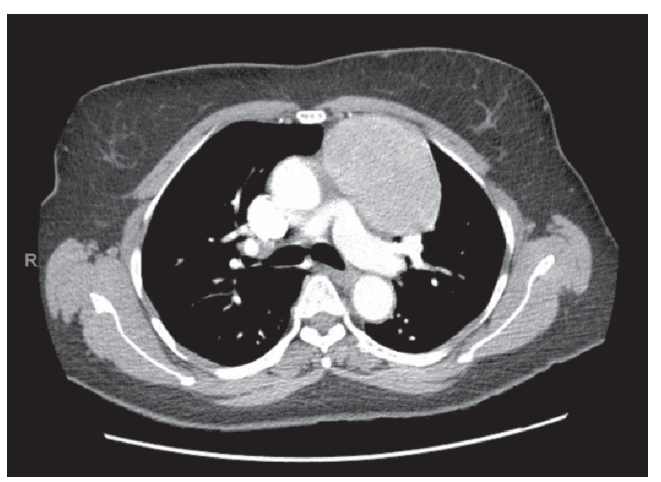

Figure 6 Large anterior mediastinal mass by CT scan of the chest. Case: a 65 -year-old female with an 8.7 -cm anterior mediastinal mass. Diagnostic evaluation revealed absence of acetylcholine receptor antibodies and normal beta-HCG and AFP levels. A robotic resection of the mediastinal mass was performed via the left chest. The pleura anterior to the mass was dissected, mobilizing the mass into the right pleural space, while moving cephalad to the level of the innominate vein. The left and right horns were dissected. Visualization and protection of the left (not involved with the mass) and right phrenic nerves was assured. Two inferior port sites were consolidated into one incision to extirpate the mass. Pathology demonstrated a type $A B$ thymoma, $9.5 \mathrm{~cm}$ in diameter. CT, computed tomography; HCG, human chorionic gonadotropin; AFP, alpha-fetoprotein.
Between 2004 and 2016, thymomas $>4$ cm were propensitymatched between robotic and sternotomy and perioperative outcomes were evaluated. The authors determined that a robotic approach was associated with lower EBL and a shorter median length of stay with similar complication rates and no difference in the rate of achieving an $\mathrm{R} 0$ resection.

Preoperatively, the surgeon must decide on the optimal side of surgery (Figure 6). For thymoma, either side is appropriate and decision-making should be based on the side of greatest tumor burden on preoperative imaging. The left sided approach is typically more difficult given the limited operative space due to the anatomy of the heart and aorta (Figure 7). For patients with myasthenia gravis, however, the left side is preferred, allowing resection of potential ectopic tissue under the left innominate vein with reduced risk of injury to the vessel. If a right sided approach is chosen, however, insertion of a port in the contralateral space can aid visualization for dissection under the left innominate vein.

Given the limited space in the mediastinum, for large masses it is helpful to open the contralateral pleural space to allow the tumor to fall away from the surgical field. This maneuver not only creates the exposure of the ipsilateral chest, but also provides countertraction of the tissues for dissection. 


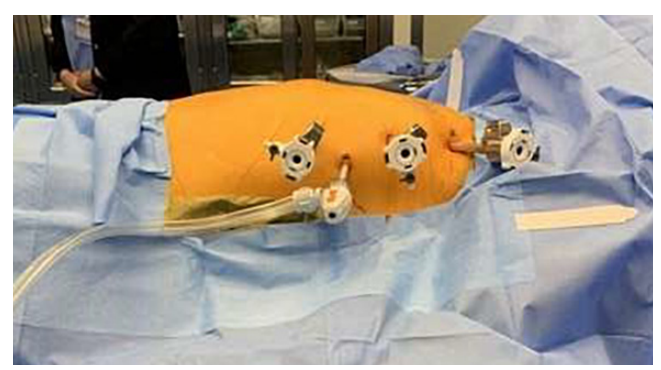

Figure 7 Left Port sites for resection of an anterior mediastinal mass. We utilize a 3-arm approach with an assistant port. The ports approximate the anterior axillary line with the assist port triangulated inferiorly.

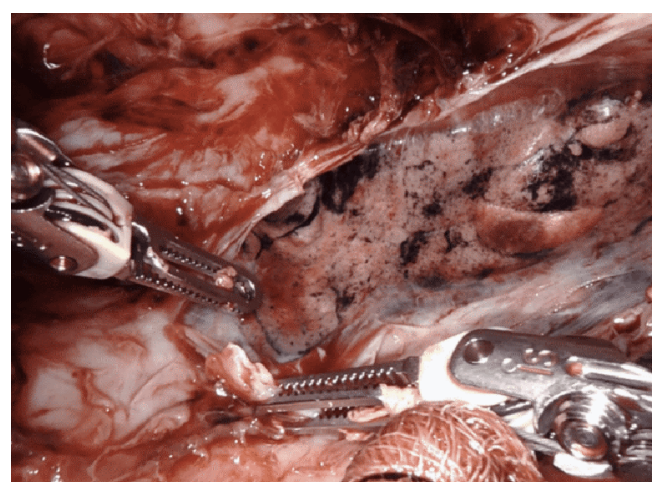

Figure 8 Robotic decortication of the left lower lobe. Case: a 51-year-old male with a history of pneumonia, persistent pleuritic chest pain, and chronic pleural effusion was brought to the operating room for robotic decortication. The lung was mobilized with clearance of multiple pockets of loculated fluid, the lung was decorticated, and the chest was drained.

\section{Decortication}

Decortication of the lung for pleural empyema or trapped lung or fibrothorax resulting from an inflammatory, hemorrhagic, or a malignant process, can be successfully performed on the robotic system. Mimicking open techniques, the robotic excels during decortication, allowing complete expansion of all surfaces of the lung. On particular advantage of the robot for decortication is the excellent optics. A high-definition, magnified, 3-dimensional view is provided from a steady, fixed perspective, and allows for the clear delineation of tissue planes such as separating the visceral pleural from the inflammatory peel. All aspects of all lobes of the lungs can be visualized on the robotic system allowing for thorough decortication to achieve pleural symphysis with complete re-expansion of the lung (Figure 8).

Creating a space for insertion of the robotic ports can be challenging, but rarely prohibitive. Initiating access into a pleural fluid cavity is often a successful strategy. We often employ a cut-down technique for insertion of the initial ports. The camera can be used to sweep adhesions from the chest wall and the remaining ports are placed under direct visualization. Carbon dioxide insufflation helps depress lung parenchyma, increasing visualization. The robot can be docked with 3 -arms until space is adequate for a $4^{\text {th }}$ port.

Establishing an appropriate tissue plane often requires bipolar cautery. The peripheral edges of the lung often allow access to the appropriate tissue layer. A rolled sponge is helpful in pushing lung parenchyma from the thickened inflammatory peel without creating rents in the visceral pleura that may lead to postoperative air leak. Intraoperative partial ventilation of the lung can help delineate thin inflammatory adhesions restricting the lung parenchyma and may help create a steadier surface for dissection than fully deflated tissue. After complete decortication, we typically leave $2-3$ chest tubes that are maintained to suction for a minimum of 48 hours.

\section{Thoracic duct ligation}

The majority of patients with high-volume chylothorax ( $>800 \mathrm{~mL} /$ day) require thoracic duct embolization or surgical thoracic duct clipping and/or ligation. A persistent, high-volume chylothorax presumes an injury of the main thoracic duct or one its proximal tributaries. Such injuries are less likely to resolve with non-interventional management strategies and delayed treatment risks leukopenia and malnutrition.

Despite the surgical approach employed (open versus minimally invasive) and provocative tests such as the preoperative administration of high fat foods or cream, a thoracic duct leak is often difficult to localize. Equally, the thoracic duct is challenging to identify due to its thin, translucent structure, frequently variable anatomy (40\%), and insidious course in the posterior mediastinum. For this reason, to assure complete ligation of the thoracic duct, if the precise location of a duct leak is unclear, operative strategy relies on mass ligation of tissues low in the posterior mediastinum.

With the high-definition, 3-dimensional view of the robotic camera, however, the thoracic duct can be clearly identified in the right chest, between the aortic and azygos vein, coursing along the lower thoracic vertebral bodies 


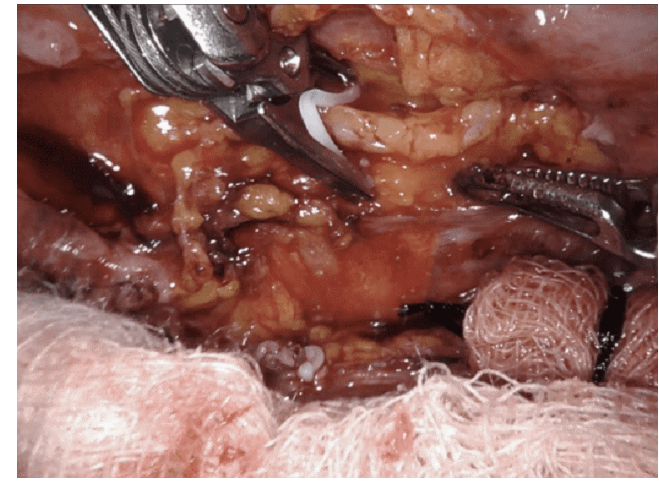

Figure 9 Right chest, clip application to the dissected thoracic duct. Case: a 67-year-old male post bilateral internal mammary artery bypass grafting complicated by bilateral high-output chylothorax. In the left chest, an avulsion of a major lymphatic duct was discovered and clipped. In the right chest, multiple small branches were clipped high in the anterior mediastinum adjacent the origin of the right internal mammary artery. The thoracic duct was localized posteriorly, clipped and ligated.

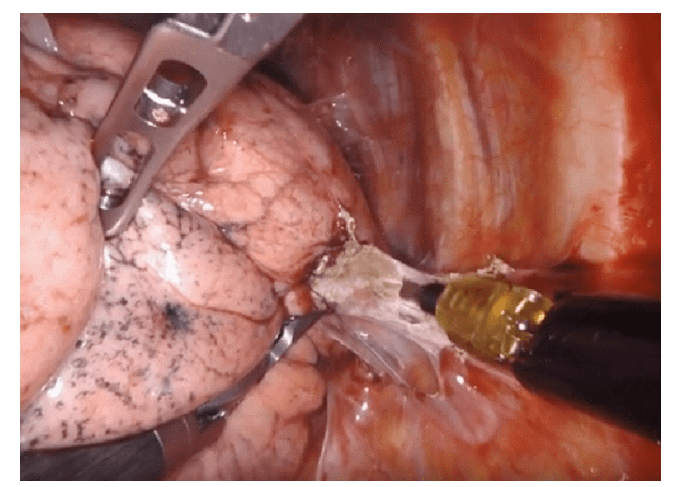

Figure 10 Robotic take-down of pleural adhesions after prior surgery and chemotherapy. Case: a 70-year-old female with a history of prior thoracotomy with left upper lobectomy followed by adjuvant chemotherapy, was found to have an enlarging left lower lobe lesion on surveillance imaging. The nodule was localized using navigational bronchoscopy with indocyanine green contrast and a non-anatomic wedge resection was performed. A limited lymphadenectomy was completed given prior lymph node dissection.

(Figure 9). Once dissected, the duct is skeletonized and mobilized. Our preference for ligation is clipping above and below the ductal defect followed by sharp transection. Attention must be given to avulsed collateral branches, which are ligated individually. The site of injury should be compulsively evaluated for persistent chyle leak. If multiple branches are encountered, or the significant inflammation obscures the field, surgical sealants can be applied widely. A mechanical pleurodesis is performed post ligation to obliterate the pleural space and chest tubes are placed for drainage. In 2008, Thompson and colleagues published the initial report of a robotic approach to thoracic duct ligation (12). A number of case reports and surgical videos have since followed, describing successful ligation on the robotic system.

\section{Resection of intralobar sequestration}

Pulmonary sequestration presents in adulthood rarely, usually with recurrent pulmonary infections, and even more rarely with hemoptysis. Ultimately, a CT scan is obtained demonstrating the aberrant vessel arising from the descending thoracic/abdominal aorta and entering the lung parenchyma, often with an inflammatory conglomerate. Surgery remains the mainstay of treatment, and often performed through a posterolateral thoracotomy, and/or video assisted thoracic surgery. These pleural cavities are often filled with adhesions or have hostile fissures from chronic inflammation. In addition, the aberrant feeding vessel of the aorta can be challenging to dissect and ligate in the face of chronic inflammation.

Use of the surgical robotic platform for resection of pulmonary sequestrations has been previously published. The largest case series, by Melfi and colleagues, four left pulmonary intralobar sequestrations were resected safely using the robotic surgical system (13). The aberrant vessel is often found within the inferior pulmonary ligament, and can be difficult to access with the diaphragm tented up. We prefer a completely portal thorascopic approach, and use $\mathrm{CO}_{2}$ insufflation allowing for the diaphragm to be pushed away. This affords direct visualization to identify the take off the descending thoracic aorta. The three-dimensional high definition imaging in conjunction with increased degrees of freedom allows for exceptional resolution to identify, isolate and ligate the aberrant arterial supply safely, and complete the pulmonary resection without unexpected avulsion and hemorrhage.

\section{Robotic surgery after prior therapy}

Dissection of tissue after a prior therapy-surgery, chemotherapy, immunotherapy, or radiation—can be difficult due to the inflammatory reaction leading to fibrotic scarring and formation of tissue adhesions (Figure 10). Adherence of 


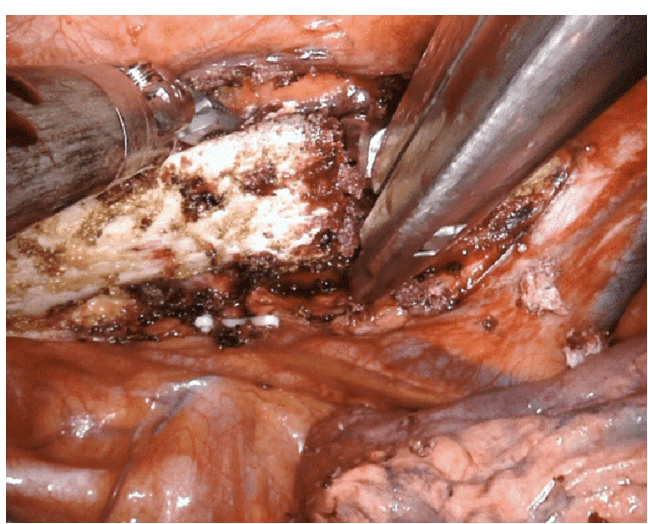

Figure 11 Ronguer division of the first rib from a robotic approach. Case: a 22-year-old male presented with refractory right arm swelling with paresthesias and diagnosed with right subclavian venous thrombosis. The patient underwent robotic resection of the right 1 st rib with division of the scalene muscles.

the lymph nodes to the hilar vessels, essentially obliterating the space between structures, can make individual skeletonization and ligation of the pulmonary artery and vein branches extremely difficult. Certain therapies may also result in increased tissue vascularity increasing the risk of operative blood loss, which can obstruct the surgical field. The timing of surgery after induction therapy is debated, although 4-6 weeks seems to be standard. In our experience, the further the patient is from therapy, the more difficult the dissection. Anecdotally, surgery after prior robotic resection results in minimal pleural adhesions. If present, adhesive disease after robotic surgery is typically limited to the port sites.

Immunotherapy as a neoadjuvant treatment strategy for patients with non-small cell lung cancer (NSCLC) has shown promise in recent trials (14). Pulmonary resection after immunotherapy can be challenging due to the development of mediastinal fibrosis, pneumonitis, and/ or pleural scaring. Dense fibrosis of the hilum or lymph node stations may obscure tissue planes or make structures adherent. A limited number of institutional series have established the safety and feasibility of minimally invasive pulmonary resection after immunotherapy, despite a high rate of conversion to open thoracotomy of $20-54 \%$.

The largest series of pulmonary resection after immunotherapy was published by Bott et al., describing 22 patients with primary or metastatic lung malignancies who were treated with nivolumab, an anti-PD-1 antibody (15). A minimally invasive approach was performed in 15 patients with only one conversion to open thoracotomy. Surgical complications occurred in $32 \%$ of the patients, the most common of which was prolonged air leak. There were no mortalities at 30 and 90 days. The 2-year overall and disease-free survival were $77 \%$ and $42 \%$ respectively. This initial experience was followed by a second study in 21 patients, all with NSCLC, who were treated with two cycles of nivolumab at two and four weeks pre-operatively (16). Of the 13 minimally invasive procedures, $54 \%$ were converted to open due to dense, vascularized hilar or mediastinal adhesions.

The optimal timing of surgical intervention after neoadjuvant immunotherapy has not been well defined. In the previously mentioned trials, patients were scheduled for resection approximately 3-5 weeks after completion of induction therapy. Delays in this treatment schedule were rate, although a small number of patients had a protracted time to surgery, in part due to immunotherapy toxicities. The rationale for a relatively expedient resection is based on the premise of a transition from inflammation to fibrosis over time.

\section{Rib resection}

First rib resection can be performed on the robotic platform, avoiding incisions high in the chest and/or neck. Most commonly indicated for thoracic outlet syndrome, first rib resection has been shown to be feasible and safe on a robotic platform. After cautery dissection of the target rib, there are a number of techniques that can be employed to excise the bone. Most involve introduction of an external instrument through a robotic port. For example, division of the rib can be achieved robotically with a bone scalpel, thoracoscopic spine drill, Kerrison, or Ronguer instrument (Figure 11). A Gigli saw can be used between two robotic graspers for a completely robotic approach, however, this requires circumferentially dissection of the rib, which must be done diligently to avoid injury to the subclavian vessels.

In a single institution series, Gharagozloo and colleagues performed robotic first rib resection in 88 patients with Paget-Schroetter syndrome (17). The technique employed involved dissection of the first rib, disarticulation at the costosternal joint, and division of the scalene muscles. The majority of patients $(57 / 83)$ had a patent subclavian vein on postoperative venogram. However, 27 patients had persistent postoperative subclavian vein occlusion requiring either angioplasty [21] or a venous stent [6] to achieve complete vein patency. At a 24-month median follow-up, 
all patients had an open subclavian vein (100\% patency rate). Importantly, the authors advise that the specimen be limited to the rib directly under the subclavian vessels with disarticulation of the rib at the costosternal junction.

\section{Conclusions}

The use of robotics in thoracic surgery continues to expand both in surgeon experience, case volume, and complexity pathology. Establishing a robotic program requires dedication from surgeons, the operative team, and institutional support. The learning curve in robotics requires dedication, but can be achieved in a step-wise approach. The robotic system offers advanced technologies that provide excellent visualization and dissection for engaging complex pathologies in the chest. Robotic technologies will continue to evolve, providing surgeons with advanced tools to address difficult cases safely and efficiently.

\section{Acknowledgments}

We appreciate collaboration from our excellent bedside physician assistants during the preparation of this manuscript: Irina Komm, Kaitlyn Booth, and Tami Healy. Funding: None.

\section{Footnote}

Provenance and Peer Review: This article was commissioned by the Guest Editor (Ghulam Abbas) for the series "Robotic Thoracic Surgery" published in Fournal of Thoracic Disease. The article has undergone external peer review.

Conflicts of Interest: All authors have completed the ICMJE uniform disclosure form (available at http://dx.doi. org/10.21037/jtd-2019-rts-05). The series "Robotic Thoracic Surgery" was commissioned by the editorial office without any funding or sponsorship. Dr. RJC reports personal fees from AstraZeneca, personal fees from Bard Davol, personal fees from Bovie Medical, personal fees from C-SATS, personal fees from ConMed, personal fees from Covidien/Medtronic, personal fees from Ethicon, personal fees from Fruit Street Health, personal fees from Google/Verb Surgical, personal fees from Intuitive Surgical, personal fees from KCI/Acelity, personal fees from Myriad Genetics, personal fees from Neomend, personal fees from Pinnacle Biologics, personal fees from ROLO-7, personal fees from Tego, personal fees from TransEnterix, outside the submitted work. Dr. CB reports personal fees from CSA Medical, outside the submitted work. Dr. MDZ reports personal fees from Intuitive Surgical, outside the submitted work. The other authors have no other conflicts of interest to declare.

Ethical Statement: The authors are accountable for all aspects of the work in ensuring that questions related to the accuracy or integrity of any part of the work are appropriately investigated and resolved.

Open Access Statement: This is an Open Access article distributed in accordance with the Creative Commons Attribution-NonCommercial-NoDerivs 4.0 International License (CC BY-NC-ND 4.0), which permits the noncommercial replication and distribution of the article with the strict proviso that no changes or edits are made and the original work is properly cited (including links to both the formal publication through the relevant DOI and the license). See: https://creativecommons.org/licenses/by-nc-nd/4.0/.

\section{References}

1. Oh DS, Reddy RM, Gorrepati ML, et al. Robotic-Assisted, Video-Assisted Thoracoscopic and Open Lobectomy: Propensity-Matched Analysis of Recent Premier Data. Ann Thorac Surg 2017;104:1733-40.

2. Cerfolio RJ, Bryant AS, Minnich DJ. Starting a robotic program in general thoracic surgery: why, how, and lessons learned. Ann Thorac Surg 2011;91:1729-36; discussion 1736-7.

3. Baldonado JJAR, Amaral M, Garrett J, et al. Credentialing for robotic lobectomy: what is the learning curve? A retrospective analysis of 272 consecutive cases by a single surgeon. J Robot Surg 2019;13:663-9.

4. Zhang Y, Liu S, Han Y, et al. Robotic Anatomical Segmentectomy: An Analysis of the Learning Curve. Ann Thorac Surg 2019;107:1515-22.

5. Feczko AF, Wang H, Nishimura K, et al. Proficiency of Robotic Lobectomy Based on Prior Surgical Technique in The Society of Thoracic Surgeons General Thoracic Database. Ann Thorac Surg 2019;108:1013-20.

6. Cao C, Cerfolio RJ, Louie BE, et al. Incidence, Management, and Outcomes of Intraoperative Catastrophes During Robotic Pulmonary Resection. Ann Thorac Surg 2019;108:1498-504.

7. Jiao W, Zhao Y, Qiu T, et al. Robotic Bronchial Sleeve 
Lobectomy for Central Lung Tumors: Technique and Outcome. Ann Thorac Surg 2019;108:211-8.

8. Ma Z, Dong A, Fan J, et al. Does sleeve lobectomy concomitant with or without pulmonary artery reconstruction (double sleeve) have favorable results for non-small cell lung cancer compared with pneumonectomy? A meta-analysis. Eur J Cardiothorac Surg 2007;32:20-8.

9. Geraci TC, Ferrari-Light D, Wang S, et al. Robotic Sleeve Resection of the Airway: Outcomes and Technical Conduct Using Video Vignettes. Ann Thorac Surg 2020;110:236-40.

10. Spaggiari L, Galetta D. Pneumonectomy for lung cancer: a further step in minimally invasive surgery. Ann Thorac Surg 2011;91:e45-7.

11. Kneuertz PJ, Kamel MK, Stiles BM, et al. Robotic Thymectomy Is Feasible for Large Thymomas: A Propensity-Matched Comparison. Ann Thorac Surg 2017;104:1673-8.

12. Thompson KJ, Kernstine KH, Grannis FW Jr, et al.

Cite this article as: Geraci TC, Scheinerman J, Chen D, Kent A, Bizekis C, Cerfolio RJ, Zervos MD. Beyond the learning curve: a review of complex cases in robotic thoracic surgery. J Thorac Dis 2021;13(10):6129-6140. doi: 10.21037/jtd-2019-rts-05
Treatment of chylothorax by robotic thoracic duct ligation. Ann Thorac Surg 2008;85:334-6.

13. Melfi FM, Viti A, Davini F, et al. Robot-assisted resection of pulmonary sequestrations. Eur J Cardiothorac Surg 2011;40:1025-6.

14. Herzberg B, Campo MJ, Gainor JF. Immune Checkpoint Inhibitors in Non-Small Cell Lung Cancer. Oncologist 2017;22:81-8.

15. Bott MJ, Cools-Lartigue J, Tan KS, et al. Safety and Feasibility of Lung Resection After Immunotherapy for Metastatic or Unresectable Tumors. Ann Thorac Surg 2018;106:178-83.

16. Bott MJ, Yang SC, Park BJ, et al. Initial results of pulmonary resection after neoadjuvant nivolumab in patients with resectable non-small cell lung cancer. J Thorac Cardiovasc Surg 2019;158:269-76.

17. Gharagozloo F, Meyer M, Tempesta B, et al. Robotic transthoracic first-rib resection for Paget-Schroetter syndrome. Eur J Cardiothorac Surg 2019;55:434-9. 